\title{
CIC-7/Ostm1 contribute to the ability of tea polyphenols to maintain bone homeostasis in C57BL/6 mice, protecting against fluorosis
}

\author{
BING-YUN LI ${ }^{1,2}$, YAN-HUI GAO ${ }^{1,2}$, JUN-RUI PEI ${ }^{1,2}$, YAN-MEI YANG ${ }^{1,2}$, WEI ZHANG ${ }^{1,2}$ and DIAN-JUN SUN ${ }^{1,2}$ \\ ${ }^{1}$ Center for Endemic Disease Control, Chinese Center for Disease Control and Prevention, Harbin Medical University; \\ ${ }^{2}$ Key Laboratory of Etiology and Epidemiology, Education Bureau of Heilongjiang Province \\ and Ministry of Health (23618504), Harbin, Heilongjiang 150081, P.R. China
}

Received June 7, 2016; Accepted February 21, 2017

DOI: 10.3892/ijmm.2017.2933

\begin{abstract}
Epidemiological investigations indicate that certain ingredients in tea bricks can antagonize the adverse effects of fluoride. Tea polyphenols (TPs), the most bioactive ingredient in tea bricks, have been demonstrated to be potent bone-supporting agents. $\mathrm{ClC}-7$ is known to be crucial for osteoclast (OC) bone resorption. Thus, in this study, we investigated the potential protective effects of TPs against fluorosis using a mouse model and explored the underlying mechanisms with particular focus on ClC-7. A total of 40, healthy, 3-week-old male C57BL/6 mice were randomly divided into 4 groups ( $n=10 /$ group) by weight as follows: distilled water (control group), $100 \mathrm{mg} / \mathrm{l}$ fluoridated water (F group), water containing $10 \mathrm{~g} / 1 \mathrm{TPs}$ (TP group) and water containing $100 \mathrm{mg} / \mathrm{l}$ fluoride and $10 \mathrm{~g} / 1$ TPs ( $\mathrm{F}+\mathrm{TP}$ group). After 15 weeks, and after the mice were sacrificed, the long bones were removed and bone marrow-derived macrophages were cultured ex vivo in order to perform several experiments. OCs were identified and counted by tartrate-resistant acid phosphatase (TRAP) staining. The consumption of fluoride resulted in severe fluorosis and in an impaired OC function [impaired bone resorption, and a low mRNA expression of nuclear factor of activated T-cells 1 (NFATc1), ATPase $\mathrm{H}^{+}$transporting V0 subunit D2 (ATP6v0d2) and osteopetrosis-associated transmembrane protein 1 (Ostm1)]. In the $\mathrm{F}+\mathrm{TP}$ group, fluorosis was attenuated and $\mathrm{OC}$ function was restored, but not the high bone fluoride content. Compared with the F group, mature
\end{abstract}

Correspondence to: Dr Dian-Jun Sun, Center for Endemic Disease Control, Chinese Center for Disease Control and Prevention, Harbin Medical University, 157 Baojian Road, Harbin, Heilongjiang 150081, P.R. China

E-mail: hrbmusdj@163.com

Key words: fluorosis, tea polyphenols, osteoclasts, bone marrowderived macrophages, chloride channel-7, osteopetrosis-associated transmembrane protein 1
OCs in the $\mathrm{F}+\mathrm{TP}$ group expressed higher mRNA levels of ClC-7 and Ostm1; the transportation and retaining of $\mathrm{Cl}^{-}$was improved, as shown by the fluorescence intensity experiment. On the whole, our findings indicate that TPs mitigate fluorosis in C57BL/6 mice by regulating OC bone resorption. Fluoride inhibits OC resorption by inhibiting $\mathrm{ClC}-7$ and Ostm1, whereas TPs attenuate this inhibitory effect of fluoride.

\section{Introduction}

Fluorosis, a disease caused by excessive fluoride intake through water and food, has been documented repeatedly in many field investigations and community surveys in a number of countries worldwide, such as Africa, China, Germany, India, United Republic of Tanzania and the US (1). In China, fluorosis includes 3 types: water-type fluorosis, coal-burning-type fluorosis and brick tea-type fluorosis. Excessive exposure to fluoride can lead to disruptions in bone metabolism and enamel development, causing skeletal fluorosis and dental fluorosis, respectively. Dental fluorosis is a less serious disease that causes a chalk-like discoloration of teeth with unsightly mottled spots or lines on tooth enamel. However, skeletal fluorosis is a crippling disease that causes pain and damage to bones and joints (2). Moreover, there is no established strategy to date for the treatment of patients with skeletal fluorosis. Thus, it is crucial to to obtain a better understanding of the mechanisms regulating the pathogenesis of fluorosis for the prevention of the potential health hazards of fluoride.

The characteristic pathological change associated with fluorosis is the acceleration of bone turnover, in which osteoclasts (OCs) play a critical role (3). An OC is a type of bone cell that disassembles and digests the composite of hydrated protein and mineral by secreting acid and collagenase, a process known as bone resorption. OCs play a critical role in the maintenance, repair and remodeling of bones. Monocytes/macrophages are recognized as OC precursors. Monocyte/macrophage precursors undergo 3 stages during their differentiation into functionally mature OCs. First, monocyte/macrophage precursors become pre-osteoclasts; second, mononuclear pre-osteoclastss fuse together to become non-functional multi-nucleated OCs expressing tartrate-resistant 
acid phosphatase (TRAP) and lacking ruffled membranes (RM); finally, non-functional multi-nucleated OCs are fully developed into functional OCs which secrete $\mathrm{H}^{+}$and enzymes (4-8). This differentiation process is regulated by various factors. Nuclear factor of activated T-cells 1 (NFATc1), a master regulator of osteoclastogenesis, regulates a number of OC-specific genes, such as TRAP, cathepsin $\mathrm{K}$ and ATPase $\mathrm{H}^{+}$transporting V0 subunit D2 (ATP6v0d2), which are known to be involved in bone resorption. It has been demonstrated that NFATc1 binds directly to the promoter regions and induces the expression of fusion-mediating molecules, such as ATP6v0d2, thereby regulating OC multi-nucleation (9). In mature OCs, ATP6v0d2, as a component of V-type $\mathrm{H}^{+}$-ATPase, mediates the acidification of the resorption lacuna, which is a prerequisite for the secretion of enzymes $(10,11)$. The efficient acidification of the resorption lacuna requires the neutralizing current through chloride channel-7 (ClC-7). $\mathrm{ClC}-7$ is a $\mathrm{Cl}^{-} / \mathrm{H}^{+}$antiporter, that resides mainly in late endosomes, lysosomes and RM of multi-nucleated OCs (12-14). The stability of ClC-7 depends on its association with osteopetrosis-associated transmembrane protein 1 (Ostm1), and Ostm1 serves as a $\beta$-subunit to support the function of ClC-7 $(15,16)$. It has been demonstrated that the loss of function of $\mathrm{ClC}-7$ or of its association with Ostm1 in humans and mice result in osteopetrosis (12). Thus, ClC-7 is crucial for OC bone resorption. Our previous study reported that fluoride decreased OC bone resorption via the inhibition of NFATc1 (45); however, to date, it has not been determined whether ClC-7 in OCs is involved in the pathogenesis of fluorosis.

The brick tea-type fluorosis caused by the consumption of fluoride from drinking tea, particularly brick tea compressed by green tea or black tea. It is a public health concern in the Northwest China, where minorities drink brick tea as drinking water. It is estimated that 31 million individuals in that region are affected (17). In addition, it is considered that the dental fluorosis detection rate in children and the skeletal fluorosis detection rate in areas affected by the brick tea-type fluorosis are higher than the rates in areas affected by water type-fluorosis (18); however, a lower number of patients with severe dental fluorosis and disabilities were found in areas affected by the brick tea-type fluorosis. Thus, it is worthy to explore whether some ingredients in brick tea can antagonize the adverse effects of fluoride. It is proposed that oxidative stress plays a critical role in the pathogenesis of fluorosis $(19,20)$. Tea polyphenols (TPs), the most bio-active ingredient in brick tea (21), have received considerable attention due to ther antioxidant effects and they have been shown to exert beneficial effects on health, including maintaining bone mass $(22,23)$. Nevertheless, the potential effect of TPs on fluorosis and the underlying mechanisms have not yet been elucidated. The role of OCs in skeletal fluorosis has attracted increased attention $(24,25,45)$. TPs have been demonstrated to be potent bone-supporting agents. Therefore, the present study aimed to investigate the potential protective effects of TPs against fluorosis using a mouse model and to explore the underlying mechanisms with particular focus on ClC-7.

\section{Materials and methods}

Animals and treatments. A total of 40 healthy, 3-week-old male C57BL/6 mice (Vital River Laboratories Animals Technology Co., Ltd., Beijing, China) were allowed to acclima- tize to an environmentally controlled room with a 12 -h light/dark cycle in an animal care facility for 7 days before beginning the experiment. Subsequently, these mice were randomly divided into 4 groups (n=10/group) by weight as follows: the distilled water (control group), $100 \mathrm{mg} / \mathrm{l}$ fluoridated water (F group), water containing $10 \mathrm{~g} / 1 \mathrm{TP}$ (TP group) and water containing $100 \mathrm{mg} / \mathrm{l}$ fluoride and $10 \mathrm{~g} / \mathrm{l} \mathrm{TP}$ (F + TP group). The animals were allowed to drink the experimental water for 15 weeks. Sodium fluoride (guaranteed reagent) was purchased from Tianjin Guangfu Fine Chemical Research Institute (Tianjin, China). TPs with a purity $>98 \%$ were purchased fromZhengzhou Chengda Chemical Products Co., Ltd. (Zhengzhou, China), and the catechins account for approximately $70 \%$ of the extractable solids. Distilled water mixed with TPs and/or fluoride and was prepared fresh daily. Body weights were monitored weekly throughout the treatment period. At the end of the experiment, all mice were euthanized by ether asphyxiation. The 4 legs were aseptically removed and dissected free of adherent soft tissue without disarticulation at the joint. All animal procedures were approved by the Animal Care and Use Committee of Harbin Medical University, Harbin, China.

Dentalfluorosisphenotyping. The dental fluorosis status of each mouse was evaluated and scored by 2 independent examiners based on the following modified Dean Index $(26,27)$ (Fig. 1A).

Assessment of skeletal fluorosis. To measure bone mineral density, the humerus bones of the mice were excised and cleaned of soft tissue, leaving the growth plates intact. The humerus bones were then examined by X-ray film using a Digital X-Ray Specimen System 4000 Pro (Kodak, Rochester, NY, USA). The integral optical density (IOD) of each X-ray film was analyzed using Image-Pro Plus software to reflect bone mineral density, as previousy described (28).

Determination of bone fluoride content. Femur bone samples were collected and cleaned by removing the soft tissues, splitting open and removing all marrow with cold PBS. The specimens were dried for $4 \mathrm{~h}$ at $105^{\circ} \mathrm{C}$ and were then weighed. Prior to ashing, the bones were heated in a smoke-free environment in a fume hood. The bones were then ashed for $6 \mathrm{~h}$ at $550^{\circ} \mathrm{C}$ and weighed again, dissolved in $1 \mathrm{~N} \mathrm{HCl}$, and neutralized by the addition of $1 \mathrm{~N} \mathrm{NaOH}$. A standard method with the ion-specific F electrode (Shanghai Weiye Instrument Plant, Shanghai, China) was used to determine the $\mathrm{F}^{-}$concentration of each dissolvent in the Institute for Endemic Fluorosis Control, Center for Endemic Disease Control, Chinese Center for Disease Control and Prevention (Harbin Medical University).

In situ bone OC numbers. The long bones were transected at a distance from the knee joints to avoid damaging the joint. Following the removal of the soft tissues, the joints were fixed in $10 \%$ formaldehyde for 7 days. The formalin-fixed tissues were then decalcified in 5\% EDTA for 1 month prior to paraffin-embedding. The paraffin-embedded specimens were then sectioned $(6 \mu \mathrm{m})$. The slides were stained using the TRAP staining kit (Shanghai Sunbio Co., Shanghai, China) and counterstained with hematoxylin. The number of $\mathrm{TRAP}^{+}$cells was counted across 4 fields of view just under the growth plate of each bone section by light microscopy at $\mathrm{x} 20$ magnification 
in an Olympus BX-51 microscope (Olympus Corp., Tokyo, Japan) and digital images were captured. Data were reported as the mean number of $\mathrm{TRAP}^{+}$cells within the 4 fields per bone section as the formation of OCs.

Assessment of OC ultrastructure in the transmission electron microscope. The metaphyses were fixed in $2.5 \%$ glutaraldehyde for 4 weeks at $4^{\circ} \mathrm{C}$ and the fixed tissues were decalcified in $10 \%$ EDTA for 4 weeks. The specimens were post-fixed for $2 \mathrm{~h}$ in $2 \%$ aqueous $\mathrm{OsO}_{4}$, dehydrated in acetone, and embedded in Epon (Electron Microscopy Sciences, Hatfield, PA, USA). Ultrathin sections were cut from blocks and then stained with uranyl acetate and lead citrate. The specimens were observed under an H-7650 transmission electron microscope (Hitachi, Ltd., Tokyo, Japan), as previously described (29,30). The cell surface structures, facing matrix, were defined as RM, an area with multiple folding of the cell membrane and clear zone $(\mathrm{CZ})$, attachment of the cell to the bone surface.

Immunohistochemistry. The sections were treated with $0.3 \% \mathrm{H}_{2} \mathrm{O}_{2}$ in ethanol to inactivate endogenous peroxidase. The sections were digested with $0.2 \%$ Triton (Amresco, LLC, Solon, OH, USA), blocked with 5\% BSA (SABC kit, Wuhan Boster Biological Technology, Ltd., Wuhan, China), incubated for $2 \mathrm{~h}$ with a 1:100 dilution of anti-rabbit ClC-7 antibody (ab86196; Abcam, Cambridge, UK) at $37^{\circ} \mathrm{C}$, treated with a biotinylated anti-rat antibody, and DAB chromogenic liquid (SABC kit). The sections were counterstained with hematoxylin.

Osteoclastogenesis. Bone marrow-derived macrophages (BMMs) were prepared as described previously $(4,31)$ and osteoclastogenesis was induced by receptor activator of $\mathrm{NF}-\kappa \mathrm{B}$ ligand (RANKL) and macrophage colony-stimulating factor (M-CSF). The femora and tibiae were aseptically removed and dissected free of adherent soft tissue. The bone ends were removed and the marrow cavity was flushed out into a Petri dish by slowly injecting $\alpha$-MEM at one end of the bone using a sterile $5 \mathrm{ml}$ syringe. The bone marrow suspension was passed repeatedly through a glass pipette to obtain a single cell suspension. The bone marrow cells were then resuspended in $\alpha$-MEM, incubated for $24 \mathrm{~h}$, and plated in 6-well plates with thye addition of $5 \mathrm{ng} / \mathrm{ml} \mathrm{M}-\mathrm{CSF}$ (Peprotech, Inc., Rocky Hill, NJ, USA) to deplete the stromal cells of cell preparations. Non-adherent cells were replated in 12-well plates at a density of $2 \times 10^{6}$ cells/well for extracting DNA and protein or in 96-well plates at a density of $1 \times 10^{5}$ cells/well in $\alpha$-MEM for OC identification, containing $30 \mathrm{ng} / \mathrm{ml} \mathrm{M-CSF}$ for 3 days to obtain the BMMs. Each group included 5 parallel wells. The BMMs were incubated with $30 \mathrm{ng} / \mathrm{ml} \mathrm{M-CSF}$ and $100 \mathrm{ng} / \mathrm{ml}$ RANKL (Peprotech, Inc.) for another 4 days which were named day 1 to 4 accordingly. The medium was replaced every 2 days.

OCs were identified and stained using the TRAP staining kit (Shanghai Sunbio Co.) on days 3 and 4. TRAP ${ }^{+}$ multinucleated cells were counted as total OCs ( $\geq 3$ nuclei) or giant OCs ( $\geq 20$ nuclei). Data are presented as the means \pm SD.

Determination of NFATcl and ATP6vOd2 mRNA expression in OCs in vitro. Gene expression in the cells was assessed by quantitative PCR (qPCR). The induced cells on day 4 were dissolved in TRIzol reagent and total RNA was extracted using the total isolation kit (Takara Bio, Dalian, China). Firststrand cDNA was synthesized using $1 \mu \mathrm{g}$ total RNA with the PrimeScript RT reagent kit with gDNA Eraser according to the manufacturer's instructions (Takara Bio). qPCR was performed on the Real-Time Fluorescence PCR Instrument (Bio-Rad Laboratories, Inc., Hercules, CA, USA) using SYBR-Green chemistry. The following commercial available primer were used for PCR: GAPDH (forward, 5'-CTGACGTGCCGCCT GGAGAAAC-3', and reverse, 5'-CCCGGCATCGAAGGTGG AAGAGT-3'), NFATc1 (forward, 5'-CTGCAA CGGGAAACG GAAGAGAAG-3', and reverse, 5'-TAT ACACCCCCAGA CCGCATAGC-3'), ATP6v0d2 (forward, 5'-ACAGACGCGC TTTAATCATCACTC-3', and reverse, 5'-ATCCСССТTCC TTTCCTCAATAAC-3') and Ostm1 (forward, 5'-GGGGAG AAACAAGGACAAACACAA-3'; reverse, 5'-ATACCCAAA CACTCCTGCCTTCCT-3'). The relative mRNA expression levels were calculated based on threshold cycle number $(\mathrm{Ct})$ and normalized to GAPDH using the comparative $\mathrm{Ct}$ method.

Western blot analysis. The cells were lysed in RIPA buffer (Beyotime Institute of Biotechnology, Shanghai, China) and protein concentrations of cellular extract were measured using an enhanced BCA Protein Assay kit (Beyotime Institute of Biotechnology). An automated capillary-based simple western system (Simon; ProteinSimple, Santa Clara, CA, USA) was then used to quantify the protein levels of $\mathrm{ClC}-7$. Compared to the traditional western blot analysis, automation allows for the more accurate and reproducible assessment of protein levels, as previously demonstrated (32). In brief, $16 \mathrm{ng}$ of protein samples were used for analysis. The proteins were immunoprobed using anti-ClC-7 antibody (ab86196; Abcam) or anti-GAPDH antibody (G9545; Sigma, St. Louis, MO, USA). Other reagents were using Simon-Rabbit (15-150 kDa) Master kit provided by ProteinSimple (San Jose, CA, USA). For quantitative analysis, the $\mathrm{ClC}-7$ signals were normalized against GAPDH.

Chloride permeation. $\mathrm{Cl}^{-}$permeation was measured using the fluorescent $\mathrm{Cl}^{-}$indicator, $N$-ethoxycarbonylmethyl-6-methoxy quinolinium bromide (MQAE), as previously described (33). The mechanism of detection is diffusion-limited collisional quenching of MQAE fluorescence. The degree of fluorescence quenching in MQAE indicates an increase in the $\mathrm{Cl}^{-}$concentration. OCs were loaded with $6 \mathrm{mmol} \cdot \mathrm{l}^{-1} \mathrm{MQAE}$ in a chloride-free buffer containing $140 \mathrm{mmol} / 1 \mathrm{NaNO}_{3}, 5 \mathrm{mmol} / 1 \mathrm{KNO}_{3}$, $5 \mathrm{mmol} / \mathrm{l}$ 4-(2-hydroxyethyl)-1-piperazineethanesulfonic acid (HEPES), $1 \mathrm{mmol} / 1 \mathrm{Mg}\left(\mathrm{NO}_{3}\right)_{2}$ and $5 \mathrm{mmol} / 1$ glucose for $60 \mathrm{~min}$ at $37^{\circ} \mathrm{C}$. Just before detection, the culture buffer was changed to a chloride buffer of similar composition with $\mathrm{Cl}^{-}$as the substituting anion. At the very moment, OCs were exposed to excitation light for 20 times at an interval of $5 \mathrm{sec}$ on the stage of an inverted microscope (Nikon Diaphot; Nikon, Tokyo, Japan) and the digitized image was recorded. The original fluorescence intensity was measured by changing from a chloride buffer to chloride-free buffer at time zero. The mean relative intensity was calculated by dividing the fluorescence intensity by the original fluorescence, as previously described (34).

Statistical analysis. All experiments were repeated 5 times, and representative results are presented. Data are presented 


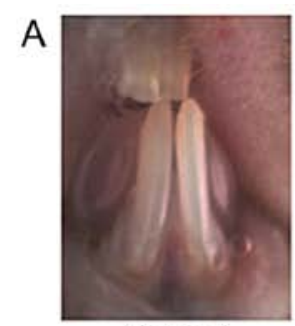

Normal

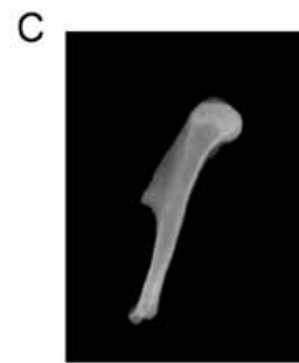

Control

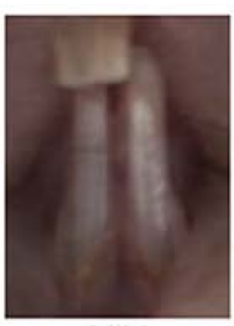

Mild

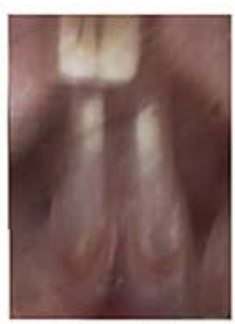

Moderate

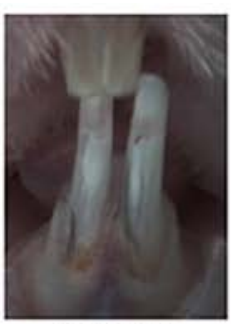

Severe
B

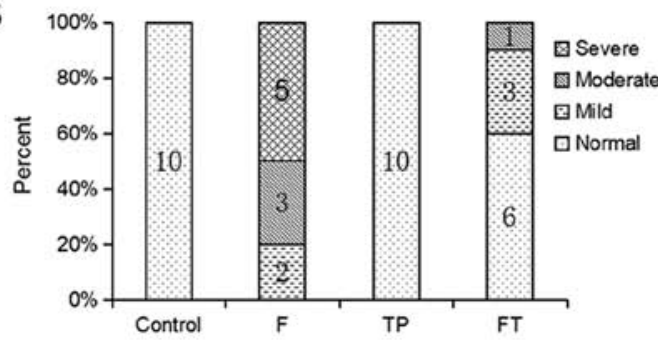

D

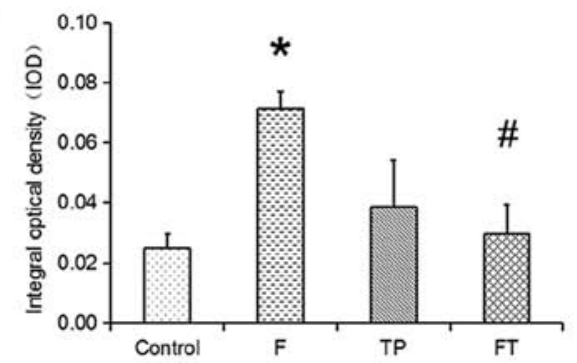

Figure 1. Fluorosis identification. (A) Representative images of each classification. Dental fluorosis in mice was classified by the modified Dean index (19,20) into 4 parts. Normal, the surface is smooth, glossy and usually a faint yellow color and is translucent; mild, the surface is smooth, glossy and usually a faint yellow color and is translucent; moderate, the white opaque areas in the enamel of the teeth are more extensive; severe, teeth often present a chalk-like appearance and fluorosed enamel with discrete or confluent pitting. (B) Incidence of dental fluorosis. The incidence of fluorosis in the groups administered fluoride was higher than the control group $(\mathrm{P}<0.05)$. The incidence of fluorosis in the $\mathrm{F}+\mathrm{TP}(\mathrm{FT})$ group was lower than that in the $\mathrm{F}$ group $(\mathrm{P}<0.05)$. $(\mathrm{C}) \mathrm{Representative} \mathrm{X}$-ray images of the humerus in each group. (D) Integral optical density (IOD) of humerus by X-ray film. ${ }^{*} \mathrm{P}<0.05$ vs. control; ${ }^{*} \mathrm{P}<0.05$ vs. F group.

as the means \pm SD and analyzed using SPSS 16.0 software. A value of $\mathrm{P}<0.05$ was considered to indicate a statistically significant difference. The normality of distribution and homogeneity of variance were tested. Dental fluorosis among the 4 groups was analyzed using the Chi-square test. The IOD of the humerus bone, $\mathrm{TRAP}^{+}$cells in vivo and in vitro, the relative mRNA expression of 4 genes, and $\mathrm{ClC}-7$ protein levels in vitro were analyzed by two-way ANOVA followed by Fisher's LSD post hoc test to evaluate the effects of fluoride, TPs or their combination. Linear by linear association was used to evaluate the association between the fluorescence intensity and detection time.

\section{Results}

Body weight and bone fluoride. Neither fluoride nor TP significantly affected the body weights of the mice throughout the study period (data not shown). The fluoride content in long bone was detected to reflect the difference of fluoride accumulation among the groups. The data indicated that the bone fluoride content in the F group $(4098.64 \pm 58.84 \mathrm{mg} / \mathrm{kg})$ and $\mathrm{F}+\mathrm{TP}$ group $(3855.70 \pm 140.77 \mathrm{mg} / \mathrm{kg})$ was significantly higher than that in the control group $(876.26 \pm 14.46 \mathrm{mg} / \mathrm{kg})(\mathrm{P}<0.001$ and $\mathrm{P}=0.002$, respectively); however, there was no difference between the $\mathrm{F}$ group and $\mathrm{F}+\mathrm{TP}$ group. The fluoride content in the TP group $(970.27 \pm 17.05 \mathrm{mg} / \mathrm{kg})$ was similar to that of the control group (876.26 $\pm 14.46 \mathrm{mg} / \mathrm{kg}$ ) (data not shown).

Dental fluorosis. Compared with the mice receiving distilled water or TPs, the mice receiving fluoride ( $\mathrm{F}$ and $\mathrm{F}+\mathrm{TP}$ groups) had a higher prevalence of dental fluorosis. From the 6 th week, the teeth of the mice in the F group were characterized by a change in color and thin white horizontal lines. At the 15th week, half of the mice in the F group had severely fluorosed enamel with discrete or confluent pitting. It is worth noting that the prevalence of dental fluorosis in the F + TP group was lower than that in the F group ( $\mathrm{P}<0.05$; Fig. 1B).

Skeletal fluorosis. A radiographic examination of the excised humerus bones confirmed the status of long bone growth (Fig. 1C). Marrow space, growth plate and cortial margins were apparent in the control specimens. However, the specimens in the $\mathrm{F}$ group exhibited a thickened cortial margin and a brighter performance. However, there was no apparent change in intensity in the TP group and F + TP group by the naked eye.The IOD of the humerus (Fig. 1D) in the F group was significantly higher than that in the control group $(\mathrm{P}=0.024)$. Of note, the IOD of the humerus in the $\mathrm{F}+\mathrm{TP}$ group was significantly lower than that in the $\mathrm{F}$ group $\mathrm{P}=0.033)$; there was thus an interaction between fluoride and TPs $(\mathrm{P}=0.015)$.

In situ bone $\mathrm{OC}$ numbers. $\mathrm{TRAP}^{+}$cells were detected as a measure of OC formation in situ and the majority of these cells appeared in the zone of cartilage calcification (Fig. 2A). The number of OCs was consistently elevated in all the treatment groups (Fig. 2B). Specifically, compared with the control group, the number of OCs increased 7-fold in the TP group, 5-fold in the $\mathrm{F}+\mathrm{TP}$ group and 3 -fold in the $\mathrm{F}$ group. Furthermore, the OCs in the TP group were more intense than those in the other groups by the naked eye.

$O C$ ultrastructure. Ultrastructural analyses of the proximal metaphyses of femurs were performed using a transmission electron microscope (Fig. 2C). In the sections from the control group and TP group, the OCs with well-developed. RM and $\mathrm{CZ}$ were frequently attached to the bone and these features 

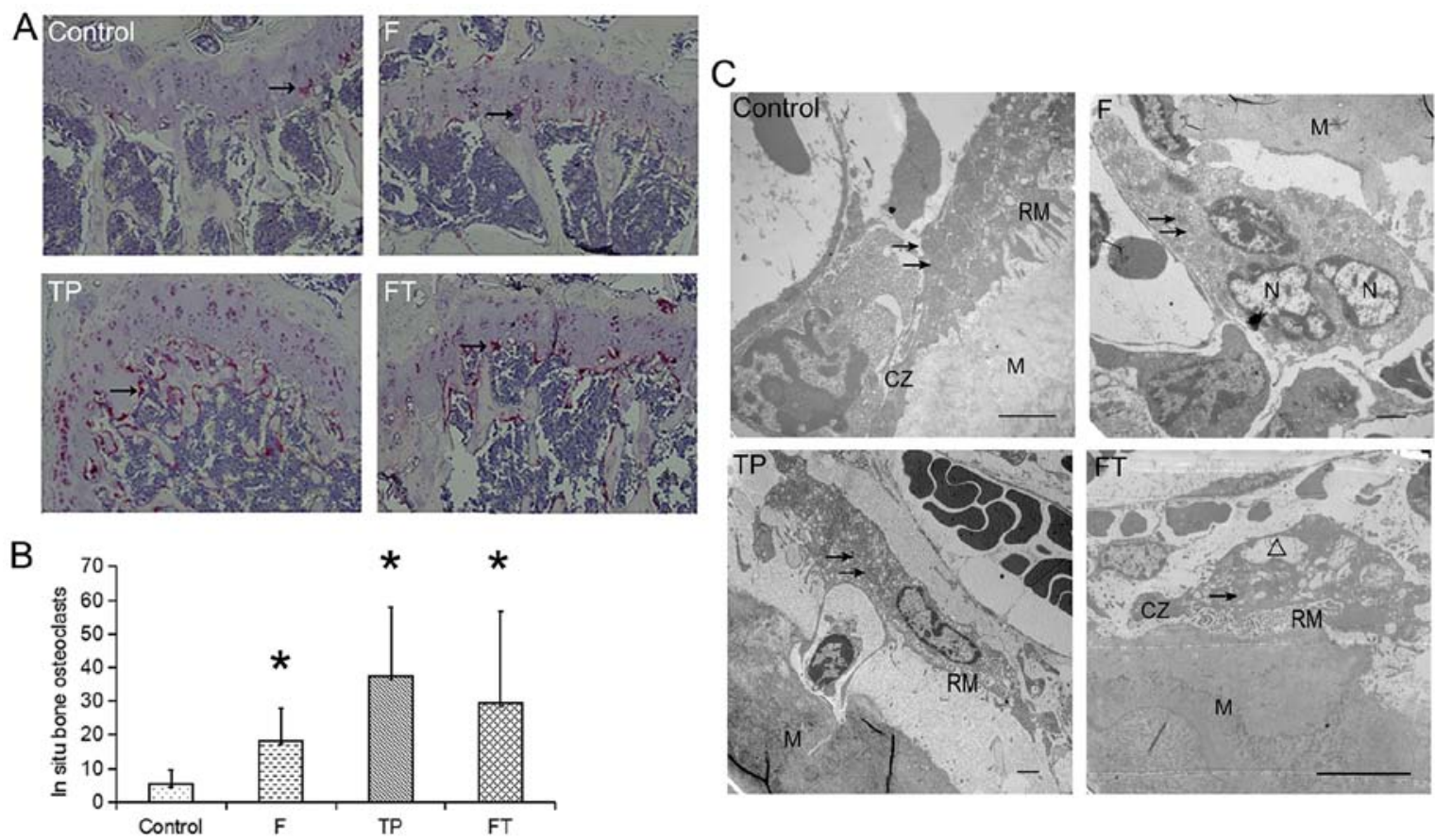

Figure 2. In situ bone osteoclasts. (A) Representative images of osteoclasts under the growth plate. Osteoclasts are indicated by black arrows. Magnification, x200. (B) Bar graphs of osteoclast numbers. ${ }^{*} \mathrm{P}<0.05$ vs. control. (C) Representative images of ultrastructural osteoclasts under the growth plate. In sections from the control group, osteoclasts with well-developed RM and CZ frequently attached to the bone matrix (M). Many vesicles containing electron-dense material (arrows) can also be seen in osteoclasts. Osteoclasts in the F + TP (FT) group exhibited vacuolation degeneration $(\Delta)$. Scale bar, $2 \mu \mathrm{m}$ (control, F and TP groups); and $10 \mu \mathrm{m}$ (FT group). RM, ruffled membrane; CZ, clear zones.

were considered to reflect capacity of bone resorption. Many vesicles containing electron-dense material (arrows) were also observed in the OCs. By contrast, a large proportion of OCs in the F group lacked the morphological signs of proper polarization, e.g., RM and CZ. Instead, the OCs exhibited a matrix-facing area with no characteristic cell surface structures, which can be considered as an immature developed cell. Moreover, the OCs in F + TP group exhibited vacuolation degeneration.

Immunohistochemistry of $\mathrm{ClC}-7$ protein in long bone. Immunohistochemical staining of $\mathrm{ClC}-7$ was performed on proximal metaphyses of femurs (Fig. 3). In the control group, ntense immunostaining was observed in the connecting zone of cartilage calcification and ossification. The location of ClC-7 protein was similar among the 4 experimental groups.

OC potential. To examine the effects of fluoride and/or TPs on $\mathrm{OC}$ formation, $\mathrm{TRAP}^{+}$multi-nucleated OCs were observed in all groups (Fig. 4). The morphology of the OCs was similar among the 4 groups (Fig. 4A). On day 3, the number of OCs (Fig. 4B) in the TP and F + TP groups was significantly higher than the control group $(\mathrm{P}=0.031$ and $\mathrm{P}<0.001$, respectively) and the number in the $\mathrm{F}+\mathrm{TP}$ group was significantly higher than that in the $\mathrm{F}$ group $(\mathrm{P}=0.023)$. On day 3 , the number of giant OCs (Fig. 4C) in the TP and F + TP groups was significantly higher than the control group $(\mathrm{P}=0.014$ and $\mathrm{P}=0.002$, respectively) and the number in the $\mathrm{F}+\mathrm{TP}$ group was also significantly higher than that in the $\mathrm{F}$ group $(\mathrm{P}=0.030)$. These differences were evident until day 4 . On day 4 , the number of giant OCs in the 4 groups increased, compared with that at day 3. Of note, the number of giant OCs in the control group was significantly lower than the other 3 groups.

mRNA expression of NFATc1, ATP6vOd2 and Ostm1 in $O C s$ in vitro. We examined the effect fluoride and/or TP administration on the mRNA expression of NFATc1 and ATP6v0d2 (Fig. 4D). The expression of NFATc1 and ATP6v0d2 in the F group was significantly lower than that in the control group $(\mathrm{P}=0.043$ and $\mathrm{P}=0.039$, respectively). The mRNA of both genes in the F + TP group was significantly higher than that in the F group (NFATc1, P=0.009; ATP6v0d2, $\mathrm{P}=0.002$ ).

mRNA expression of Ostm1 in OCs in vitro. Ostm1 mRNA expression in the F group decreased almost 600-fold compared to the control group (Fig. 5A). The mRNA expression of Ostm1 in the F + TP group was significantly higher than that in the $\mathrm{F}$ group (Ostm1, $\mathrm{P}=0.033)$.

ClC-7 expression in OCs in vitro. Giant OCs were regarded as having absorbing capacity, and thereafter the protein level of $\mathrm{ClC}-7$ on day 4 was detected (Fig. $5 \mathrm{~B}$ and $\mathrm{C}$ ). The protein level of $\mathrm{ClC}-7$ in the $\mathrm{F}$ group $(0.08 \pm 0.02)$ was significantly lower than that in the control group $(0.15 \pm 0.02, \mathrm{P}=0.006)$. ClC-7 protein expression levels in the TP group $(0.24 \pm 0.02)$ and $\mathrm{F}+\mathrm{TP}$ group $(0.16 \pm 0.05)$ were both significantly higher than the level in the $\mathrm{F}$ group $(\mathrm{P}=0.012$ and $\mathrm{P}=0.03$, respectively). There was thus an interaction between fluoride and TPs $(\mathrm{P}=0.029)$.

Fluorescence intensity in OCs in vitro. The ability of $\mathrm{ClC}$ in OCs to transport $\mathrm{Cl}^{-}$in all groups was detected on day 4 (Fig. 5D). 

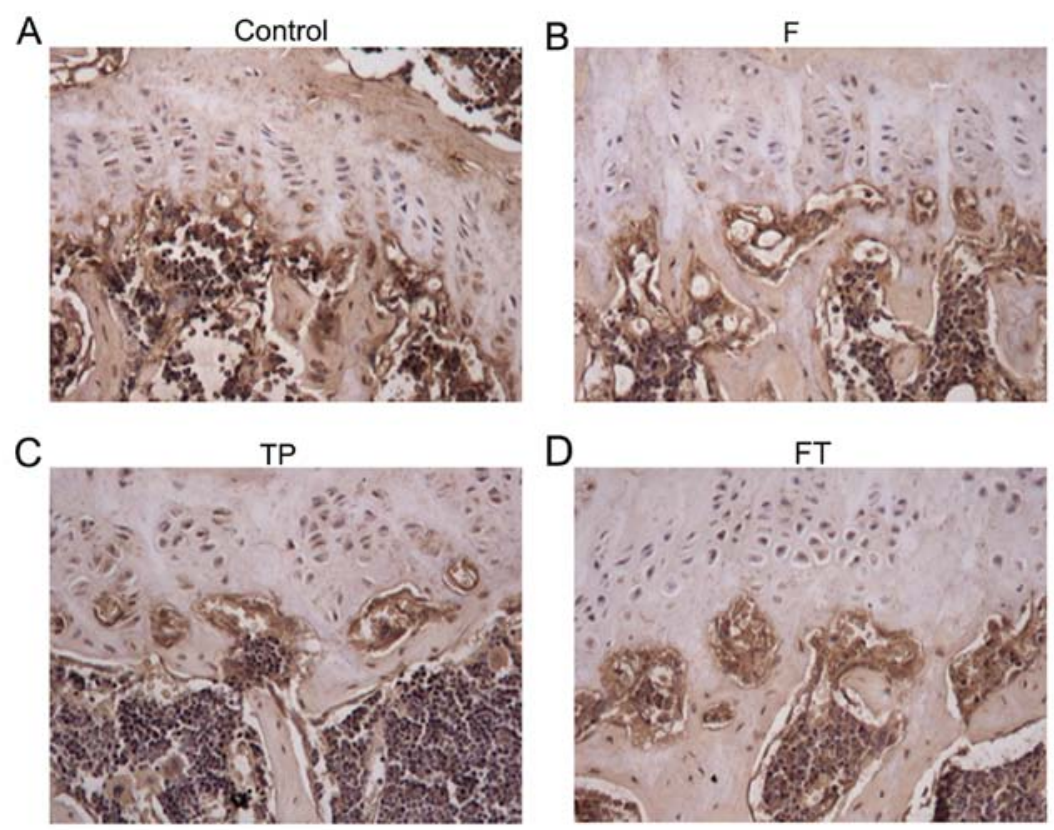

Figure 3. Immunohistochemical staining of ClC-7. Representative images of osteoclasts under the growth plate of the (A) control, (B) fluoride (F), (C) tea polyphenol (TP); and (D) fluoride and tea polyphenol (F + TP) (FT) groups. Location of protein expression of ClC-7 was similar among the 4 experimental groups. Magnification, $\mathrm{x} 200$

A

Day 3

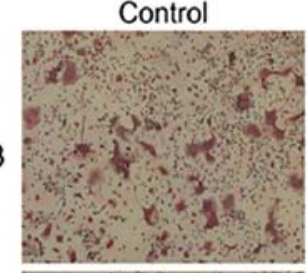

Day 4

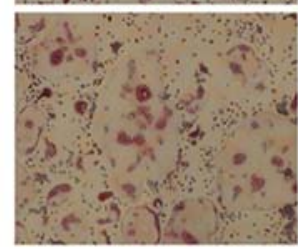

B
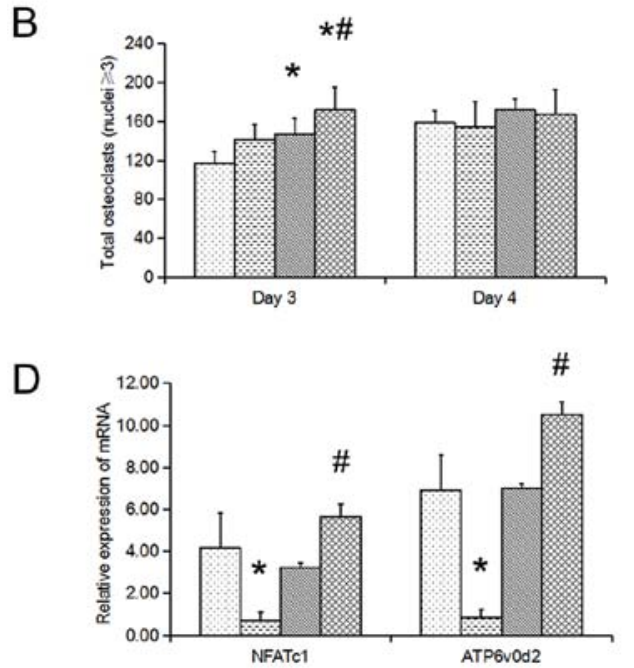

$\mathrm{F}$
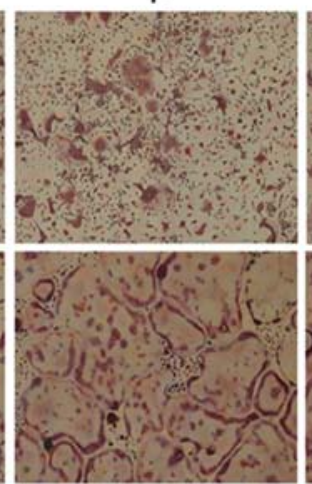

C
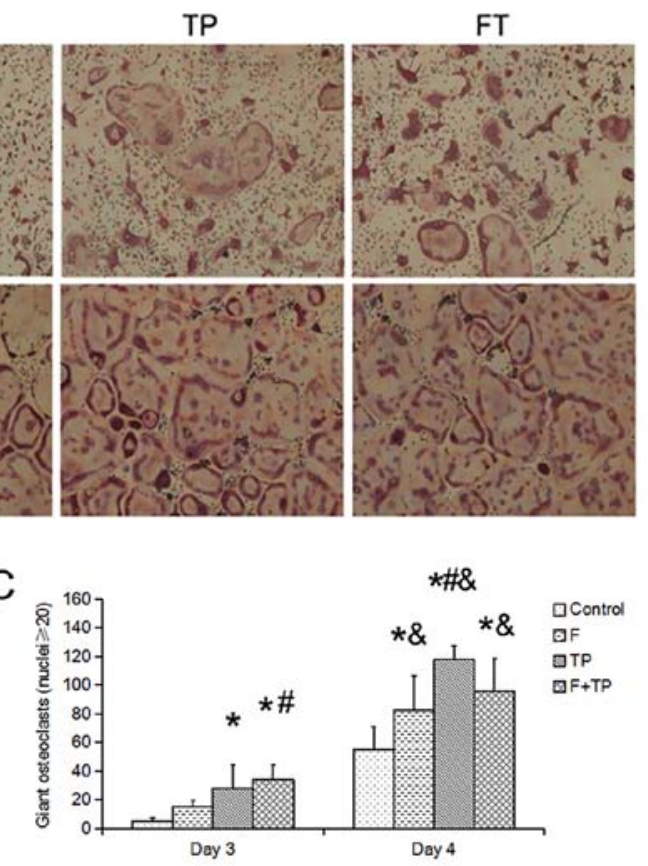

DControl

DF

DTP

Figure 4. Osteoclast formation and function-related gene mRNA expression. (A) Formation of osteoclasts differentiated from BMMs isolated from mice. Morphology of osteoclasts was similarly among the 4 groups. (B and C) Osteoclasts were counted as total osteoclasts ( $\geq 3$ nuclei) or giant osteoclasts ( $\geq 20$ nuclei). Data are presented as the means \pm SD. (D) Giant osteoclasts were generally considered as mature osteoclasts. NFATc1 and ATP6v0d 2 mRNA expression on day 4 was determined. ${ }^{*} \mathrm{P}<0.05$ vs. control; ${ }^{\#} \mathrm{P}<0.05$ vs. F group; ${ }^{\&} \mathrm{P}<0.05$ vs. the same group on day 3 . F, fluoride group; TP, tea polyphenol group; FT, fluoride and tea polyphenol group; BMMs, bone marrow-derived macrophages. 
A

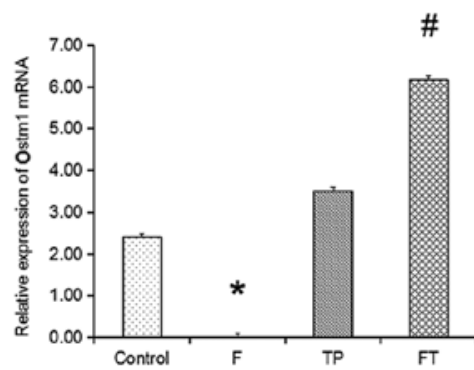

C

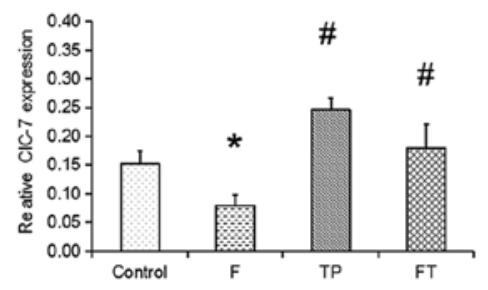

B
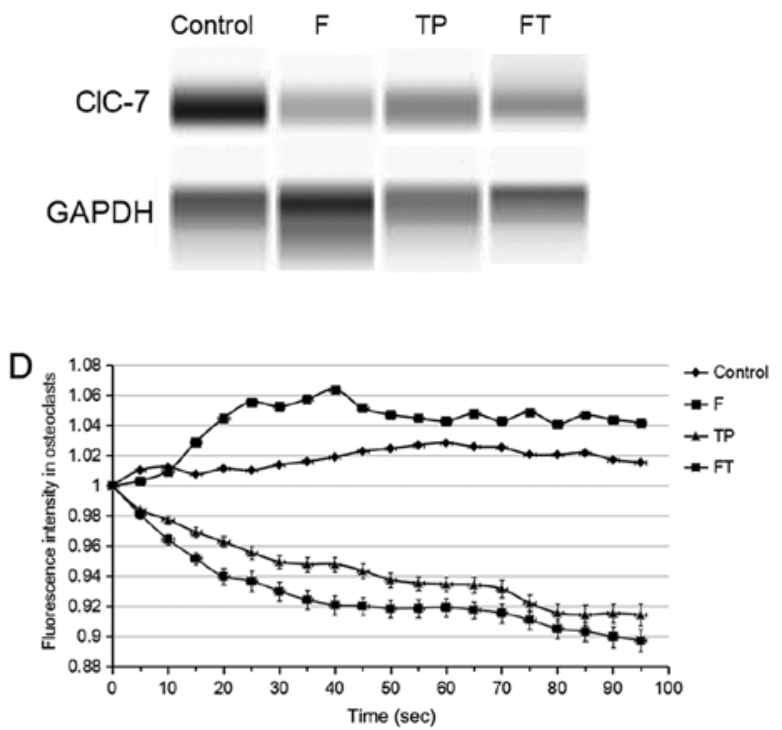

Figure 5. Effect of ClC-7/Ostm1 in osteoclasts. (A) Ostm1 mRNA expression in osteoclasts was determined on day 4 . (B) ClC-7 (87 kDa) and GAPDH (42 kDa) were detected by western blot analysis. (C) The relative $\mathrm{ClC}-7$ expression in the 4 groups. ${ }^{*} \mathrm{P}<0.05$ vs. control; ${ }^{*} \mathrm{P}<0.05$ vs. $\mathrm{F}$ group. (D) The ability of $\mathrm{ClC}$ transport $\mathrm{Cl}^{-}$among all groups was reflected by detecting the fluorescence intensity with osteoclasts on day 4 . The degree of fluorescent quenching in $\mathrm{MQAE}$ indicates increase in $\mathrm{Cl}^{-}$concentration. Mean relative intensity was calculated by dividing each fluorescence intensity by the original fluorescence. $\mathrm{F}$, fluoride group; TP, tea polyphenol group; FT, fluoride and tea polyphenol group; Ostm1, osteopetrosis-associated transmembrane protein 1.

Within $90 \mathrm{sec}$, the fluorescence intensity was continuously declining in the TP and $\mathrm{F}+\mathrm{TP}$ groups $(\mathrm{P}<0.001$ and $\mathrm{P}=0.024$, respectively), suggesting that $\mathrm{Cl}^{-}$could be accumulated in OCs in these groups; however, the fluorescence intensity in the $\mathrm{F}$ group exhibited an increasing trend $(\mathrm{P}<0.001)$, suggesting that $\mathrm{OCs}$ in the $\mathrm{F}$ group did not retain $\mathrm{Cl}^{-}$.

\section{Discussion}

The skeletal fluorosis phenotype of the murine model and human disease is characterized by a combination of osteosclerosis, osteomalacia and osteoporosis in varying degrees. The low bone mass in C57BL/6 mice appears to be sensitive to the effects of fluoride on bone cells (35-39). Therefore, in the present study, C57BL/6 mice were utilized to investigate the potential protective effects of TPs against chronic fluorosis. In the $\mathrm{F}$ group, the mice had a higher prevalence of dental fluorosis and severe fluorosed enamel with discrete or confluent pitting. A radiographic examination of excised humerus bones revealed a thickened cortial margin and significantly higher IOD values. Such results were in agreement with those of another study using female C57BL/6 mice (35), indicating that fluorosis model was successfully constructed. The bone fluoride content remained consistent between the F group and $\mathrm{F}+\mathrm{TP}$ group, suggesting that TP supplementation did not affect the accumulation of fluoride in the bone. However, compared to the F group, the F + TP group exhibited a significant mitigation of fluorosis, as shown by the prevalence of dental fluorosis and IOD value of the humerus, suggesting that TPs exerted a beneficial effect on bone homeostasis, protecting against the toxic effects of fluoride.

In our mouse model, the consumption of $100 \mathrm{mg} / \mathrm{l}$ fluoride stimulated bone formation. Bone remodeling is a complex, yet coordinated process that is responsible by two main types of cells, osteoblasts and OCs. Fluoride, as an anabolic agent, is capable of promoting osteoblast proliferation, whereas the influence of fluoride on OCs remains controversial. In our study, $100 \mathrm{mg} / \mathrm{l}$ fluoride increased the number of $\mathrm{TRAP}^{+}$cells in the region below the growth plate, suggesting that fluoride stimulated the formation of OCs, which is consistent with a previous report using the $\mathrm{C} 3 \mathrm{H} / \mathrm{HeJ}(\mathrm{C} 3 \mathrm{H})$ inbred mouse strain with $50 \mathrm{mg} / 1$ fluoride for 3 weeks (37). However, ultrastructural analysis revealed that the OCs in the F group exhibited an underveloped RM and $\mathrm{CZ}$, and failed to attach to the bone matrix, which is consistent with previous animal and cell studies $(40,41)$. It is known that OCs resorb bone by establishing a circle between themselves and the bone surface, and that the formation and development of specific membrane structures, such as RM and CZ, are important for bone resorption (42). Moreover, patients with defective acidification have increased numbers of OCs with decreased bone resorption $(43,44)$. Therefore, our results suggested that fluoride increased the number of OCs; however, these OCs exhibited defective acidification and could not resorb bone matrix. Compared to the the F group, mice in F + TP group had a greater number of $\mathrm{TRAP}^{+}$cells in the region below the growth plate, and most OCs exhibited a well-developed RM and CZ, suggesting that TP alleviated defective acidification induced by fluoride. OCs are terminally differentiated multi-nucleated cells derived from mononuclear hematopoietic cells of the monocyte/macrophage lineage in bone marrow. RANKL and M-CSF are essential and sufficient for osteoclastogenesis from BMMs. Thus, primary BMMs isolated from mouse long bones are utilized as OC precursors to generate mature OCs ex vivo. In this study, we isolated primary BMMs from differently treated mice to observe the effects of fluoride and/or TPs on OC formation and 
for ex vivo osteoclastogenesis assays. We noted that fluoride had little effect on osteoclatogenesis; this result was similar to that of our previous study (45); the number of OCs ( $>3$ nuclei) or the number of giant OCs ( $>20$ nuclei) in mice in the F + TP group was greater than that in the $\mathrm{F}$ group on day 3 , suggesting that TPs promoted osteoclastogenesis in association with exposure to high levels of fluoride.

Altough the molecular mechanisms by which fluoride regulates bone resorption have not yet been illustrated, a previous in vitro study by our group indicated that fluoride inhibited the resorption activity of OCs by inhibiting NFATc1 and its downstream genes, such as Atp6v0d2 (45). NFATc1 plays an important role in osteoclastogenesis by regulating the fusion of pre-osteoclastss and bone resorption $(8,46,47)$. Atp6v0d2 is an essential component of the OC-specific proton pump that mediates extracellular acidification in bone resorption (6). In our mouse model, we further examined the effect of fluoride and/or TPs on the expression of NFATc1 and Atp6v0d2 in new OCs at the mRNA level. Our results revealed that the expression of NFATc1 and ATP6v0d2 in the F group was significantly lower than that in the control group, which is consistent with our previous study, whereas the mRNA expression of both genes in the $\mathrm{F}+\mathrm{TP}$ group was significantly higher than that in the F group, suggesting that TP attenuated the inhibitory effect on these two genes induced by fluoride.

$\mathrm{ClC}-7$, as one member of the $\mathrm{ClC}$ family, may support bone resorption by electrically shunting the $\mathrm{H}^{+}$-ATPase that acidifies the resorption lacuna of OCs, or by facilitating the insertion of proton-pump containing vesicles into the RM. It is known to be associated with osteopetrosis, that is characterized by OC dysfunction, impaired bone resorption and poor bone remodeling (48). In osteopetrosis, OCs are developed without an RM and fail to generate an acidic bone resorption compartment or resorb bone. In our model, OCs in the F group exhibited an underveloped RM and $\mathrm{CZ}$, and failed to attach to the bone matrix; thus, we investigated the effect of fluoride and/or TPs on $\mathrm{ClC}-7$. In the present study, the $\mathrm{ClC}-7$ level in the $\mathrm{F}$ group was significantly lower than that in the control group; however, $\mathrm{ClC}-7$ expression in the $\mathrm{F}+\mathrm{TP}$ group was significantly higher than that in the $\mathrm{F}$ group, suggesting that TPs attenuated the inhibitory effect on $\mathrm{ClC}-7$ protein induced by fluoride.

ClC-7, as a $\mathrm{Cl}^{-} / \mathrm{H}^{+}$antiporter, is important for lysosomal acidification. It is the primary $\mathrm{Cl}^{-}$permeation pathway in lysosomes (15). Thus, we further detected $\mathrm{Cl}^{-}$permeation using fluorescent the $\mathrm{Cl}^{-}$indicator, MQAE (33). The degree of fluorescence quenching in MQAE indicates an increase in $\mathrm{Cl}^{-}$in cells. In the $\mathrm{F}$ group, new OCs did not retain $\mathrm{Cl}^{-}$; however, new OCs in the $\mathrm{F}+\mathrm{TP}$ group retained $\mathrm{Cl}^{-}$. These results were consistent with the change in the protein expression of ClC-7. ClC-7 can reach the lysosome and can be degraded in the lysosome due to the lack of $N$-linked glycosylation sites. Ostm1, a type I membrane protein with heavily glycosylated amino-terminal portion and a short cytoplasmic tail, shields $\mathrm{ClC}-7$ from degradation by lysosomal proteases $(15,16,49)$. Thus, we investigated the influence of fluoride and/or TPs on Ostm1 expression at the mRNA level. Our results indicated that in new OCs in the F group, Ostm1 mRNA expression was significantly lower than that in the control group; however, Ostm1 mRNA expression in the FT group was significantly higher than that in the F group, suggesting that fluoride decreased ClC-7 expression by inhibiting Ostm1; TPs, however, attenuated this effect. This may be one of the reasons as to why the severity of fluorosis in areas affected by brick tea-type fluorosis is milder than that in areas affected by water-type fluorosis. Thus, perhaps the use of TPs could be considered for the prevention of fluorosis in areas affected by water-type fluorosis.

In conclusion, in the present study, we demonstrated that TPs exerted beneficial effects against fluorosis by regulating OC resorption ability. We reported that fluoride inhibited OC resorption by decreased $\mathrm{ClC}-7$ and Ostm1 expression, whereas TPs attenuated these inhibitory effects of fluoride.

\section{Acknowledgements}

The present study was supported by the National Natural Science Foundation of China (no. 81072252).

\section{References}

1. WHO: http://www.who.int/water_sanitation_health/publications/fluoride-in-drinking-water/en/. Accessed Nov 10, 2011.

2. Wang C, Gao Y, Wang W, Zhao L, Zhang W, Han H, Shi Y, Yu G and Sun D: A national cross-sectional study on effects of fluoride-safe water supply on the prevalence of fluorosis in China. BMJ Open 2: e001564, 2012.

3. Sun D and Gao Y: Molecular mechanism of pathogenesis of osteofluorosis: a discussion in the view of bone turnover. Chin $\mathrm{J}$ Endemiol 27: 239-241, 2008.

4. Suda T, Takahashi N, Udagawa N, Jimi E, Gillespie MT and Martin TJ: Modulation of osteoclast differentiation and function by the new members of the tumor necrosis factor receptor and ligand families. Endocr Rev 20: 345-357, 1999.

5. Lacey DL, Timms E, Tan HL, Kelley MJ, Dunstan CR, Burgess T, Elliott R, Colombero A, Elliott G, Scully S, et al: Osteoprotegerin ligand is a cytokine that regulates osteoclast differentiation and activation. Cell 93: 165-176, 1998.

6. Väänänen HK, Zhao H, Mulari M and Halleen JM: The cell biology of osteoclast function. J Cell Sci 113: 377-381, 2000.

7. Väänänen HK, Karhukorpi EK, Sundquist K, Wallmark B, Roininen I, Hentunen T, Tuukkanen J and Lakkakorpi P: Evidence for the presence of a proton pump of the vacuolar $\mathrm{H}(+)$-ATPase type in the ruffled borders of osteoclasts. J Cell Biol 111: 1305-1311, 1990.

8. Boyle WJ, Simonet WS and Lacey DL: Osteoclast differentiation and activation. Nature 423: 337-342, 2003.

9. Kim K, Lee SH, Ha Kim J, Choi Y and Kim N: NFATc1 induces osteoclast fusion via up-regulation of Atp6v0d2 and the dendritic cell-specific transmembrane protein (DC-STAMP). Mol Endocrinol 22: 176-185, 2008.

10. Baron R, Neff L, Louvard D and Courtoy PJ: Cell-mediated extracellular acidification and bone resorption: Evidence for a low $\mathrm{pH}$ in resorbing lacunae and localization of a $100-\mathrm{kD}$ lysosomal membrane protein at the osteoclast ruffled border. J Cell Biol 101: 2210-2222, 1985.

11. Blair HC, Teitelbaum SL, Ghiselli R and Gluck S: Osteoclastic bone resorption by a polarized vacuolar proton pump. Science 245: 855-857, 1989.

12. Kornak U, Kasper D, Bösl MR, Kaiser E, Schweizer M, Schulz A, Friedrich W, Delling G and Jentsch TJ: Loss of the ClC-7 chloride channel leads to osteopetrosis in mice and man. Cell 104: 205-215, 2001.

13. Jentsch TJ, Stein V, Weinreich F and Zdebik AA: Molecular structure and physiological function of chloride channels. Physiol Rev 82: 503-568, 2002.

14. Zhao Q, Wei Q, He A, Jia R and Xiao Y: CLC-7: A potential therapeutic target for the treatment of osteoporosis and neurodegeneration. Biochem Biophys Res Commun 384: 277-279, 2009.

15. Leisle L, Ludwig CF, Wagner FA, Jentsch TJ and Stauber T: ClC-7 is a slowly voltage-gated $2 \mathrm{Cl}(-) / 1 \mathrm{H}(+)$-exchanger and requires Ostm1 for transport activity. EMBO J 30: 2140-2152, 2011.

16. Lange PF, Wartosch L, Jentsch TJ and Fuhrmann JC: ClC-7 requires Ostm1 as a beta-subunit to support bone resorption and lysosomal function. Nature 440: 220-223, 2006. 
17. Sun D, Gao Y, Zhao L, Yu G, Wu L and Li Q: A cross-sectional survey on drinking brick-tea type fluorosis in China. Chin J Endemiol 27: 513-517, 2008.

18. Sun D, Gao Y, Yu G, Liu Y, Zhao X, Wu L, Li Q and Sun Y: Prevalence characteristics of drink-tea type fluorosis. Chin J Endemiol 27: 121-123, 2008.

19. Varol E, Icli A, Aksoy F, Bas HA, Sutcu R, Ersoy IH, Varol S and Ozaydin M: Evaluation of total oxidative status and total antioxidant capacity in patients with endemic fluorosis. Toxicol Ind Health 29: 175-180, 2013.

20. Wang Q, Cui KP, Xu YY, Gao YL, Zhao J, Li DS, Li XL and Huang HJ: Coal-burning endemic fluorosis is associated with reduced activity in antioxidative enzymes and $\mathrm{Cu} / \mathrm{Zn}-\mathrm{SOD}$ gene expression. Environ Geochem Health 36: 107-115, 2014.

21. Cabrera C, Artacho R and Giménez R: Beneficial effects of green tea - a review. J Am Coll Nutr 25: 79-99, 2006.

22. Shen CL, Yeh JK, Cao JJ and Wang JS: Green tea and bone metabolism. Nutr Res 29: 437-456, 2009.

23. Shen CL, Yeh JK, Stoecker BJ, Chyu MC and Wang JS: Green tea polyphenols mitigate deterioration of bone microarchitecture in middle-aged female rats. Bone 44: 684-690, 2009.

24. Junrui P, Bingyun L, Yanhui G, Xu J, Darko GM and Dianjun S Relationship between fluoride exposure and osteoclast markers during RANKL-induced osteoclast differentiation. Environ Toxicol Pharmacol 46: 241-245, 2016

25. Liu XL, Song J, Liu KJ, Wang WP, Xu C, Zhang YZ and Liu Y: Role of inhibition of osteogenesis function by Sema4D/Plexin-B1 signaling pathway in skeletal fluorosis in vitro. J Huazhong Univ Sci Technolog Med Sci 35: 712-715, 2015.

26. Dean HT: Fluorine in the control of dental caries. J Am Dent Assoc 52: 1-8, 1956.

27. Dean HT: Endemic fluorosis and its relation to dental caries. 1938 Public Health Rep 121 (Suppl 1): 213-219, discussion 212, 2006.

28. Drozdzowska B, Pluskiewicz W and Tarnawska B: Panoramicbased mandibular indices in relation to mandibular bone mineral density and skeletal status assessed by dual energy X-ray absorptiometry and quantitative ultrasound. Dentomaxillofac Radiol 31 361-367, 2002.

29. Nordahl J, Andersson G and Reinholt FP: Chondroclasts and osteoclasts in bones of young rats: Comparison of ultrastructural and functional features. Calcif Tissue Int 63: 401-408, 1998.

30. Nordahl J, Hollberg K, Mengarelli-Widholm S, Andersson G and Reinholt FP: Morphological and functional features of clasts in low phosphate, vitamin D-deficiency rickets. Calcif Tissue Int 67: 400-407, 2000

31. Chambers TJ: Regulation of the differentiation and function of osteoclasts. J Pathol 192: 4-13, 2000.

32. Chen JQ, Heldman MR, Herrmann MA, Kedei N, Woo W, Blumberg PM and Goldsmith PK: Absolute quantitation of endogenous proteins with precision and accuracy using a capillary western system. Anal Biochem 442: 97-103, 2013.

33. Dragomir A and Roomans GM: Increased chloride efflux in colchicine-resistant airway epithelial cell lines. Biochem Pharmacol 68: 253-261, 2004.

34. Chen S, Wan XL and Sears M: pICln can regulate swelling-induced Cl- currents in either layer of rabbit ciliary epithelium. Biochem Biophys Res Commun 246: 59-63, 1998.
35. Yan D, Gurumurthy A, Wright M, Pfeiler TW, Loboa EG and Everett ET: Genetic background influences fluoride's effects on osteoclastogenesis. Bone 41: 1036-1044, 2007.

36. Linkhart TA, Linkhart SG, Kodama Y, Farley JR, Dimai HP, Wright KR, Wergedal JE, Sheng M, Beamer WG, Donahue LR, et al: Osteoclast formation in bone marrow cultures from two inbred strains of mice with different bone densities. J Bone Miner Res 14: 39-46, 1999.

37. Judex S, Garman R, Squire M, Donahue LR and Rubin C: Genetically based influences on the site-specific regulation of trabecular and cortical bone morphology. J Bone Miner Res, 19: 600-606, 2004

38. Turner CH, Hsieh YF, Müller R, Bouxsein ML, Baylink DJ, Rosen CJ, Grynpas MD, Donahue LR and Beamer WG: Genetic regulation of cortical and trabecular bone strength and microstructure in inbred strains of mice. J Bone Miner Res 15: 1126-1131, 2000.

39. Turner CH, Hsieh YF, Müller R, Bouxsein ML, Rosen CJ, McCrann ME, Donahue LR and Beamer WG: Variation in bone biomechanical properties, microstructure, and density in BXH recombinant inbred mice. J Bone Miner Res 16, 206-213, 2001.

40. Szewczyk KA, Fuller K and Chambers TJ: Distinctive subdomains in the resorbing surface of osteoclasts. PLoS One 8: e60285, 2013.

41. Wu H1, Xu G and Li YP: Atp6v0d2 is an essential component of the osteoclast-specific proton pump that mediates extracellular acidification in bone resorption. J Bone Miner Res 24: 871-885, 2009.

42. Väänänen K: Mechanism of osteoclast mediated bone resorption - rationale for the design of new therapeutics. Adv Drug Deliv Rev 57: 959-971, 2005.

43. Taranta A, Migliaccio S, Recchia I, Caniglia M, Luciani M, De Rossi G, Dionisi-Vici C, Pinto RM, Francalanci P, Boldrini R, et al: Genotype-phenotype relationship in human ATP6i-dependent autosomal recessive osteopetrosis. Am J Pathol 162: 57-68, 2003.

44. de Vernejoul MC and Bénichou O: Human osteopetrosis and other sclerosing disorders: Recent genetic developments. Calcif Tissue Int 69: 1-6, 2001.

45. Pei J, Li B, Gao Y, Wei Y, Zhou L, Yao H, Wang J and Sun D: Fluoride decreased osteoclastic bone resorption through the inhibition of NFATc1 gene expression. Environ Toxicol 29: 588-595, 2014.

46. Teitelbaum SL: Bone resorption by osteoclasts. Science 289 1504-1508, 2000.

47. Teitelbaum SL and Ross FP: Genetic regulation of osteoclast development and function. Nat Rev Genet 4: 638-649, 2003.

48. Neutzsky-Wulff AV, Sims NA, Supanchart C, Kornak U, Felsenberg D, Poulton IJ, Martin TJ, Karsdal MA and Henriksen K: Severe developmental bone phenotype in ClC-7 deficient mice. Dev Biol 344: 1001-1010, 2010.

49. Plans V, Rickheit $\mathrm{G}$ and Jentsch TJ: Physiological roles of $\mathrm{CLC} \mathrm{Cl(-)/H} \mathrm{(+)} \mathrm{exchangers} \mathrm{in} \mathrm{renal} \mathrm{proximal} \mathrm{tubules.} \mathrm{Pflugers}$ Arch 458: 23-37, 2009. 